\title{
Clinical profile and colonoscopic findings in patients with lower gastrointestinal haemorrhage: a descriptive cross-sectional study
}

\author{
Subash Bhattarai ${ }^{1}$, Ramesh Raj Acharya², Bishnu Jwarchan ${ }^{3}$, Dipesh Karki ${ }^{4}$ \\ ${ }^{1}$ Associate Professor, ${ }^{2,3,4}$ Lecturer, Department of Medicine, Manipal College of Medical Sciences , Pokhara, Nepal
}

Background: Most of the bleeding in the lower gastrointestinal tract are usually located in the rectum, colon and terminal ileum. Colonoscopy is an invasive procedure used for both diagnostic and therapeutic purposes for detection of lower gastrointestinal (GI) tract pathologies and haemorrhage. Aims and Objective: The purpose of the study was to understand the clinical profile and colonoscopic findings in patients with lower gastrointestinal haemorrhage. Materials and Methods: Seventy-two patients presenting with lower GI haemorrhage were included in the study. All patients underwent colonoscopy after achieving hemodynamic stability and bowel preparation. Clinical profile and colonoscopic findings were studied. Results: The common aetiologies of lower GI haemorrhage were haemorrhoids followed by nonspecific colitis, colorectal polyp and carcinoma of colon. Rectum followed by sigmoid harbored majority of pathologies that presented with lower GI haemorrhage. Conclusions: The diagnosis of the pathological lesion and management of underlying cause not only prevents another episode of lower Gl haemorrhage but also help in reducing morbidity and mortality. Colonoscopy or at least sigmoidoscopy is strongly recommended for evaluation, diagnosis and management of lower GI haemorrhage.

Key words: Colitis; Colonoscopy; Colorectal; Gastrointestinal haemorrhage

\section{Access this article online}

Website:

http://nepjol.info/index.php/AJMS DOI: $10.3126 /$ ajms.v11i4.28718

E-ISSN: 2091-0576

P-ISSN: 2467-9100

Copyright (c) 2020 Asian Journal of Medical Sciences

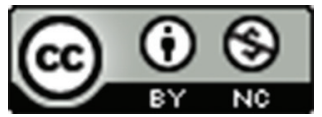

This work is licensed under a Creative Commons Attribution-NonCommercial 4.0 International License.

\section{INTRODUCTION}

Lower gastrointestinal ( GI ) haemorrhage is defined as bleeding distal to the ligament of Treitz. ${ }^{1}$ Most of the pathological lesions and haemorrhage in the lower GI tract are usually located in the rectum, sigmoid and left sided colon. ${ }^{2}$ It is usually suspected when patients complain of haematochezia (passage of frank bright red blood) or bloody diarrhea. ${ }^{3-5}$ Most of the bleeding from the lower GI tract stops spontaneously and patients usually have favorable outcomes compared to upper GI haemorrhage. However, morbidity and mortality tend to increase in older patients and with other pre existing co morbidities. ${ }^{6}$
Colonoscopy is an invasive procedure used for both diagnostic and therapeutic purpose for detection and management of the diseases of rectum, colon, and terminal ileum. Direct visualization makes it investigation of choice for colonic pathology. Mucosal biopsy alongside diagnostic purpose and minimal invasive therapeutic procedure can be done during the colonoscopy procedure.

In country like Nepal, colonoscopy is still being done for diagnostic purpose. Very limited studies are available in this part of the country regarding lower GI haemorrhage. Considering above facts this study was designed to study the clinical profiles and colonoscopic finding of the patient presenting with lower GI haemorrhage in a tertiary care 
center at Manipal Teaching Hospital in Pokhara, Gandaki Province, Nepal.

\section{MATERIALS AND METHODS}

This observational, cross sectional hospital based prospective study was carried out in the unit of Medical gastroenterology under Medicine department at Manipal College of Medical Sciences and Teaching Hospital at Pokhara, Gandaki Province, Nepal during April 2018 till March 2020 after obtaining ethical clearance from Institutional Research Committee (MEMG/IRC/333/ GA) and informed consent from patient or patient relatives.

All patients randomly admitted from emergency irrespective of age, sex, co- morbidities with lower GI haemorrhage were undertaken for the study. In emergency department, patients were first hemodynamically stabilized with intravenous (IV) fluids. Injection tranexamic acid was used for achieving haemostatis. Blood and blood products were transfused when required. History, physical examination and data considering demographic variables, symptomatolgy, clinical features, blood tests and coagulation profile were carried out. Ultrasonography of abdomen, CT abdomen was done when relevant. After hemodynamically stabilization, bowel preparation and pre medications and informed consent, colonoscopy was performed. Bowel preparation was done with 2 liters of Poly Ethylene Glycol given 8 hours prior to procedure and patient kept on liquid diet from 24 hours prior to procedure, whenever possible. In case of acute emergency, only sigmoidoscopy after rectal enema was performed. During the procedure, patient was laid on left lateral position, intravenous Hyoscine butyl bromide was given. Each patient underwent endoscopic investigation by standard flexible colonoscope (PENTAX EPK 700, PENTAX JAPAN Inc). Colonoscopy findings were noted and biopsy of tissue was sent when relevant. The lesion with recent stigmata of haemorrhage or pathological lesion was considered the cause of lower GI haemorrhage. Colonoscopy findings followed by histology of biopsies were helpful in making final diagnosis and aetiologies of lower GI bleed.

\section{Inclusion criteria}

All patients with lower GI haemorrhage.

\section{Exclusion criteria}

1. Patient with persistent hemodynamic instability.

2. Same patient re admitted and colonoscopy showing same finding in re admissions.

3. Patients with incomplete records or who fail to give informed consent.

\section{Statistical analysis}

Data were collected covering the relevant parameters for the study. All categorical data were expressed in percent and absolute number. All numerical continuous data were expressed in mean $\pm \mathrm{SD}$. The data analysis was done using Statistical Packages for the Social Sciences (SPSS) 20 (IBM Corp. Released 2011. IBM SPSS Statistics for Windows, Version 20.0. Armonk, NY: IBM Corp.)

\section{RESULTS}

A total of 83 patients underwent colonoscopy for lower GI haemorrhage between April 2018 till March 2020. But 9 patients had to be excluded because of inadequate data. Finally, a total of 72 patients that comprised of $42(60 \%)$ male and $30(40 \%)$ female were enrolled for the study. (M: $\mathrm{F}=3: 2)$. The mean age of subjects was $48 \pm 9.34$ years with a range of $18-79$ years of age.

Patients were further classified as per sex and age groups (Table 1). A total of $12(16.7 \%)$ patients presented with shock. Patients had various presentation and complaints alongside history of lower GI bleed (Table 2).

Co morbid conditions were present in $570(86.4 \%)$ patients (Table 3).

Risk factors in the study participants include non vegetarian in $72(100 \%)$, actively smoking in $64(88.9 \%)$, chronic alcohol consumption in $56(77.8 \%)$, recent (within a week) consumption of NSAIDS and steroids in 18 (25\%).

Pallor was the most common clinical finding in 58 (80.5\%), followed by abdominal tenderness in 44(61.1\%),

\begin{tabular}{|c|c|c|c|c|}
\hline \multirow[t]{2}{*}{ Sex } & \multicolumn{3}{|c|}{ Age Groups } & \multirow{2}{*}{$\begin{array}{c}\text { Total } \\
(\mathrm{N}=72\end{array}$} \\
\hline & $<40$ yrs & $40-59$ yrs & 60 years and above & \\
\hline Male & 9 & 22 & 11 & 42 \\
\hline Female & 6 & 16 & 8 & 30 \\
\hline
\end{tabular}

\begin{tabular}{lcc}
$\begin{array}{l}\text { Table 2: Complaints of patients with lower GI } \\
\text { haemorrhage }\end{array}$ & Patients & Percentage \\
\hline Presentation with & 58 & 80.5 \\
\hline Haematochezia (frank rectal bleeding) & 46 & 63.9 \\
Bloody diarrhea (blood mixed stools) & 33 & 45.8 \\
Altered bowel habits & 32 & 44.4 \\
Chronic constipation & 28 & 38.9 \\
Chronic abdominal pain & 12 & 16.7 \\
Shock & 8 & 11.1 \\
Mass felt or protrusion out of anus & & \\
during straining or defecation & &
\end{tabular}


hepatomegaly in 30(41.7\%), and splenomegaly in 12 $(16.7 \%)$ patients under study.

Mean hemoglobin at presentation was $9.9 \mathrm{gm} \%$ with a range of $4.8 \mathrm{gm} \%$ to $12.5 \mathrm{gm} \%$. Hemoglobin was $<10 \mathrm{gm} \%$ in 35 subjects. Average of 1.9 units and a maximum of 6 units of blood were transfused. Mean platelets count was $106,000 / \mathrm{mm}^{3}$ with a minimum of $40,000 / \mathrm{mm}^{3}$ and a maximum of $5,10,000 / \mathrm{mm}^{3}$.

Complete colonoscopy with visualization of terminal ileum could be performed in only $64(88.9 \%)$ patients. Terminal ileum could not be intubated in 5 patients. Scope could not be negotiated beyond hepatic flexure in another subset of 3 patients. The location of the lower GI tract lesions and the relative frequency of hemorrhagic sites has been shown in pie diagram 1.

Erosions, ulcers and hemorrhoids in the rectum were the most common findings in colonoscopy.

Etiology of bleed was not identified in six (8.3\%) patients as their colonoscopy was normal. The most common aetiologies for lower GI bleed after colonoscopy and histology were haemorrhoids and non specific colitis followed by others (Table 3).

Non specific colitis seen in 16 biopsies $(22.2 \%)$ was the most common histological finding. Colitis was more

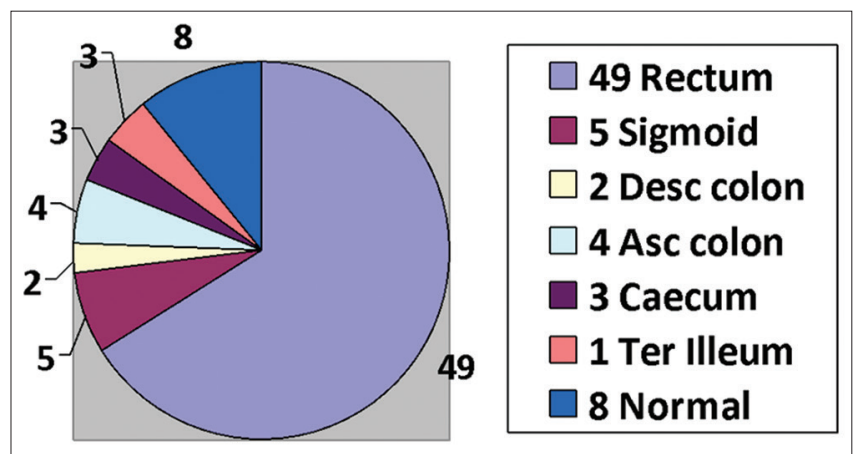

Pie diagram 1: Sites of colonic pathology on colonoscopy $(N=72)$.

\begin{tabular}{|c|c|c|}
\hline Co morbidities & Number $(\mathrm{N}=72)$ & Percentage \\
\hline Chronic liver disease & 33 & 45.6 \\
\hline chronic kidney disease & 2 & 2.8 \\
\hline Ischemic heart disease & 7 & 9.7 \\
\hline Past history of CVA & 4 & 5.6 \\
\hline COPD / Bronchial Asthma & 2 & 2.8 \\
\hline Arthritis & 3 & 4.2 \\
\hline Diabetes Mellitus & 4 & 5.6 \\
\hline Hypertension & 6 & 8.3 \\
\hline No significant past medical history & 11 & 15.3 \\
\hline Total & 72 & 100 \\
\hline
\end{tabular}

common in males (M: F $=3: 2)$ with mean age of $41 \pm 8.37$ years. Rectum was the most common site of involvement and was seen in more than $2 / 3^{\text {rd }}$ of cases.

Haemorrhoids were the most common colonoscopic finding in patients with lower GI haemorrhage and seen in $20(27.8 \%)$ patients. They were seen at mean age of $36 \pm 8.28$ with male predominance (M: $\mathrm{F}=3: 2$ ). Bleeding haemorrhoids were commonly seen in patients among 30 49 years of age group. Majority $(70 \%)$ of the haemorrhoids were of Grades II/III. Eighteen $(90 \%)$ patients with haemorrhoids gave history of bleeding per rectum at the end of defecation. All patients had history of chronic constipation.

Ulcerative colitis was the next most important colonic pathology and was seen in $14(19.4 \%)$ patients. Mean age at presentation was $34 \pm 7.65$ years with slight female predominance ( $\mathrm{M}: \mathrm{F}=3: 4)$ ). About $75 \%$ of the cases were identified in age group below 40 years of age. Only 1 patient with Crohns disease presented with lower GI haemorrhage. The most common site of bleed with ulcerative colitis was recto-sigmoid seen in $12(85.7 \%)$. Pan colitis was observed in $8(57.1 \%)$ patients.

Colorectal carcinoma was the etiology of lower GI haemorrhage in $13.9 \%$. Rectal Carcinoma was source of bleed in 6 patients. Three malignant colonic growths were detected in sigmoid colon, 1 in descending colon and 2 in ascending colon. All these 12 cases of colorectal carcinoma were histological adenocarcinoma. Mean age of presentation with colorectal carcinoma was $65 \pm 11.53$ with male predominance (M: F=3:1). Eight $(66.6 \%)$ patients were aged between $60-79$ years of age. Two cases were aged below 40 . The youngest patient was a 32-year male diagnosed with rectal carcinoma. It was observed that all these 12 patients with colorectal carcinoma were non vegetarian and all were chronic smokers

Polyps were the etiology of lower GI bleed in only 4 $(5.6 \%)$ patients. 3 large solitary pedunculated polyps were located in the rectum which were removed by snare polypectomy. Mean age of these patients was $48 \pm 9.55$ with male predominance (M: F=3:1). Three of the 4 polyp had adenomatous polyp with mild dysplasia and only $1 \mathrm{had}$ malignant adenocarcinoma.

\section{DISCUSSION}

Majority of the patients with lower GI haemorrhage were found in the age group of 40-59 years in the current study. Age groups of 51-60 years were common in the studies by Rahman et al ${ }^{8}$ in Bangladesh and Chaudhary et $\mathrm{al}^{9}$ in Nepal. 
However, a lower age group of 18-30 years was observed in an Indian study by Hajare et al. ${ }^{10}$ Elderly population (>60 years) constituted $40 \%$ of lower GI bleed by Dar et al. ${ }^{11}$ in Kashmir, India.

Mean age of presentation of lower GI bleed was $59 \pm$ 16.9 years in a European study by Fernández et al. ${ }^{12}$ The mean age of patients presenting with lower GI bleed were $41.9 \pm 15.0$ years and $43.82 \pm 17.96$ years in the studies by Rahman et al. ${ }^{8}$ and Hajare et al. ${ }^{10}$ respectively. The mean age of patients was $48 \pm 9.34$ years in the current study. Chaudhary et $\mathrm{al}^{9}$ in Western Nepal reported almost similar age group of $46.93 \pm 16.99$ years reflecting the possibility of similar environmental and etiological risk factors and similar patterns of lower GI tract diseases prevailing within the country.

All these studies including this study had male predominance. The male predominance may be attributable to gender heath seeking practices as well as more risk factors like smoking, alcoholism and unhealthy dietary habits more prevalent among Nepalese males.

Haematochezia $(80.55 \%)$ followed by bloody diarrhea $(63.9 \%)$, altered bowel habits $(45.8 \%)$, chronic constipation $(44.9 \%)$ and abdominal pain $(38.9 \%)$ were the common complaints by the patients in our study. Haematochezia $(80 \%)$ was the common presentation of lower GI haemorrhage followed by constipation $(78 \%)$ in the study by Hajare et al. ${ }^{10}$ Our finding was consistent with the study by Dar et al. ${ }^{11}$ where hematochezia followed by bloody diarrhea were the main complaints.

The most colonic pathologies presenting with lower GI haemorrhage were located in rectum in $68 \%$ in the current study. Chaudhary et $\mathrm{al}^{9}$ reported a lesser $(59.42 \%)$ of rectal pathologies with lower GI haemorrhage.

Many studies in the Western countries have identified polyp, diverticular diseases, neoplasia and IBD as the common aetiologies of lower GI haemorrhage. The common colonoscopic findings for lower GI haemorrhage were polyps $(25.1 \%)$ followed by diverticular disease $(24 \%)$, neoplasia $(12.6 \%)$, inflammatory bowel disease $(9.4 \%)$, unspecific proctitis $(2.4 \%)$ and others $(6.1 \%)$ in the study by Fernández et al. ${ }^{12}$ The common pathologies of lower GI haemorrhage were diverticulitis $(15 \%$;), inflammatory bowel disease $(13 \%)$, ischemic colitis $(11 \%)$ and haemorrhoids (11\%) in a study by Hreinsson et al. ${ }^{13}$ in Iceland. Colonic diverticulitis, haemorrhoids and polyps/ neoplasms were identified as the common sources of lower GI bleed in United Kingdom. ${ }^{14,15}$ In the study of by Alobaidi et al. ${ }^{16}$ in Iraq, the most common causes were ulcerative colitis in $31.3 \%$ patients, followed by cancer in
$21.8 \%$ patients and polyps in $15.2 \%$ patients. Whereas, in the Indian sub continent, haemorrhoids, IBD and polyps have been described as common aetiologies of lower GI bleed in various studies. But literatures vary among their orders and frequencies of these lesions. Maximum (28.5\%) patients had hemorrhoids, followed by colorectal cancer $(27.5 \%)$ in the study by Rahman et al. ${ }^{8}$ in Bangladesh. Hemorrhoids (48\%) were the commonest etiology but followed by ulcerative colitis (24\%) in the Indian study by Hazare et al. ${ }^{10}$ Growth/ polyp in $29.3 \%$ followed by IBD in $17.7 \%$ were the most common aetiologies for lower GI bleed in Kashmir, India in the study by Dar et al. ${ }^{11}$ The common colonoscopic findings in patients with lower GI haemorrhage in the study by Chaudhary et al. in western Nepal were growth $(14.49 \%)$, followed by haemorrhoids (13.04\%) and polyp (8.7\%). In another Nepalese study by Shrestha et al, ${ }^{17}$ haemorrhoids $(35.2 \%)$ followed by non-specific colitis $(24.8 \%)$ colon polyp (18.3\%), IBD (10.4\%) and colon cancer (6.5\%) were the common aetiologies of lower GI haemorrhage. Haemorrhoids (27.8\%) and non-specific colitis (25\%) were also the two common aetiologies of lower GI bleed in the current study, in consistency with the study by Shrestha et al. ${ }^{17}$ This was followed by ulcerative colitis $(22.2 \%)$, carcinoma colon $(6.9 \%)$ and polyps $(5.6 \%)$ in the current study. Compared to Western literature, diverticulosis was uncommon in the above-mentioned studies in the Indian sub-continent including ours. Shrestha et al. ${ }^{17}$ in Nepal documented $1.7 \%$ and the current study identified only $1.4 \%$ diverticulosis as etiology of lower GI haemorrhage.

Haemorrhoids were common cause of lower GI haemorrhage among 30-49 years of age by Shrestha et al. ${ }^{17}$ as well as in the current study. The most common histological diagnosis was nonspecific colitis in the study by Chaudhary et al. ${ }^{9}$ in Western Nepal and Shrestha et al ${ }^{17}$ as well as in the current study.

The first presentation of Ulcerative colitis has been found to be in $3^{\text {rd }}$ and $4^{\text {th }}$ decades in many literatures. Around $79 \%$ patients were below 40 years in the study in Iraq by Alobaidi et al. ${ }^{16}$. Shrestha et al., ${ }^{17}$ reported a majority of IBD cases including ulcerative colitis in 16-29 years of age group. In the current study, ulcerative colitis was the second most important colonic pathology for lower GI haemorrhage and was seen in $19.4 \%$ patients and at younger age group, finding similar to the other studies. About $75 \%$ of the cases were identified in age group below 40 years of age. Mean age at presentation were $27.9 \pm 6.5$ years by Shrestha et al. ${ }^{17}$ and $34 \pm 7.65$ years in the current study. The most common site for ulcerative colitis was recto sigmoid in $72.6 \%$ patients in the study by Alobaidi et al. ${ }^{16}$. The finding was similar to our study $(75.8 \%)$. Pan colitis was observed in $27.4 \%$ by Alobaidi et al. ${ }^{16}$ whereas it was $30.3 \%$ in the current study. 
Colorectal cancers were more common in rectum around $60 \%$, followed by sigmoid $25 \%$, while remainder parts of colon constitute only $14 \%$ according to the study by Alobaidi et al. ${ }^{16}$ Our study also demonstrated a similar anatomical site of distribution. Half and one fourth of malignant growth was detected in rectum and sigmoid colon respectively. Colon cancer was seen in $6.5 \%$ of the study participants and especially in age groups of 70-84 years in the study by Shrestha et al. ${ }^{17}$ Colorectal carcinoma were seen in more $(13.9 \%)$ patients in the current study and majority $(66.6 \%)$ were observed at a even younger age group of $60-79$ years. Two $(16.6 \%)$ cases of rectal carcinoma were aged below 40 years of age. With the use of alcohol, smoking at earlier ages, bad dietary habits with decrease consumption of dietary factors, the prevalence of carcinoma tends to be shifting to a decade earlier.

Colon polyps were the source of lower GI haemorrhage in $18.3 \%$ in the Nepalese study by Shrestha et al., ${ }^{17}$ whereas it constituted only $5.6 \%$ in the current study. Polyps were detected commonly in the age groups of 50-69 years in both the studies.

No source of bleeding could be identified from $1.4 \%$ and $16 \%$ in the studies by Shrestha et al. ${ }^{17}$ and Rahman et al. ${ }^{8}$ respectively. Whereas, no source of bleeding could be identified in $8.3 \%$ in the current study.

\section{CONCLUSIONS}

The common aetiologies of lower GI haemorrhage were haemorrhoids followed by non-specific colitis, colorectal polyp and carcinoma. Rectum, sigmoid and left sided colon harbor majority of the pathologies and they are common sites for lower GI bleed. Most of the bleeding from the lower GI tract stops spontaneously. The timely diagnosis of the pathological lesion and immediate management of lower GI haemorrhage helps in preventing another episode of lower GI bleed thus can be helpful in reducing morbidity and mortality. Colonoscopy or at least sigmoidoscopy is strongly recommended for evaluation, diagnosis and management of lower GI tract pathologies and haemorrhage.

\section{LIMITATION OF THE STUDY}

The study reflects to the findings of a specific geographical area with a small sample size. Due to lack of sedation, illeo ceacal intubation was not possible in a minority of cases. This was primarily due to patient refusal for further negotiation of the scope due to abdominal pain and discomfort. Etiology of lower GI bleed could not be identified in some patients because of unavailability of enteroscopy, capsule endoscopy, and other needful modalities.

\section{ACKNOWLEDGEMENT}

The authors take this opportunity to thank Endoscopy unit and its staffs at Manipal Teaching Hospital to carry out the present study.

\section{REFERENCES}

1. Zuccaro G Jr. Management of the adult patient with acute lower gastrointestinal bleeding. American College of Gastroenterology. Practice Parameters Committee. Am J Gastroenterol. 1998; 93(8):1202-1208.

https://doi.org/10.1111/j.1572-0241.1998.00395.x

2. Gralnek IM, Neeman Zand Strate LL. Acute Lower Gastrointestinal Bleeding. N Engl J Med. 2017;376(23):1054-1063.

https://doi.org/10.1056/NEJMcp1603455

3. Jensen DM and Machicado GA. Diagnosis and treatment of severe hematochezia. The role of urgent colonoscopy after purge. Gastroenterology. 1988; 95(6):1569-1574.

https://doi.org/10.1016/S0016-5085(88)80079-9

4. Zuckerman GR, Trellis DR, Sherman TM and Clouse RE. An objective measure of stool color for differentiating upper from lower gastrointestinal bleeding. Dig Dis Sci. 1995; 40(8):1614-1621. https://doi.org/10.1007/BF02212679

5. Wilcox CM, Alexander LN and Cotsonis G. A prospective characterization of upper gastro intestinal hemorrhage presenting with hematochezia. Am J Gastroenterol. 1997; 92(2):231-235.

6. Strate LL, Ayanian JZ, Kotler G and Syngal S. Risk factors for mortality in lower intestinal bleeding. Clin Gastroenterol Hepatol. 2008 ;6(9):1004-1010.

https://doi.org/10.1016/j.cgh.2008.03.021

7. Rex DK, Petrini JL, Baron TH, Chak A, Cohen J, Deal SE, et al. Quality indicators for colonoscopy. Gastrointest Endosc. 2006; 63(4):16-28.

https://doi.org/10.1016/j.gie.2006.02.021

8. Rahman MM, Bhuiyan MH, Ferdaus $\mathrm{AM}$ and Mahmud R. Correlation between Clinical Diagnosis and Colonoscopic Findings of Patients Presented with Lower Gastrointestinal Bleeding. Mymensingh Med J. 2015;24(2):238-243.

9. Chaudhary S, Khatri P, Dhakal PR, Shahi A and Jaiswal NK. Clinical Profile and Colonoscopic Findings in Patients Presented with Lower Gastrointestinal Bleeding. IOSR Journal of Dental and Medical Sciences (IOSR-JDMS). 2019;18(11):50-55.

10. Hajare $S$ and Kantamaneni R. Etiological profile of patients with lower gastrointestinal bleeding: A 1-year cross-sectional study. Arch Med Health Sci. 2018; 6:300-302.

https://doi.org/10.4103/amhs.amhs_33_17

11. Dar IA, Dar WR, Khan MA, Kasana BA, Sofi NU and Hussain M. Etiology, clinical presentation, diagnosis and management of lower gastrointestinal bleed in a Tertiary Care Hospital in India: A retro-prospective study. Journal of Digestive Endoscopy. 2015;6(03):101-109. https://doi.org/10.4103/0976-5042.165697

12. Fernández E, Linares A, Alonso JL, Sotorrio NG, Artimez ML, Giganto $\mathrm{F}$, et al. Colonoscopic findings in patients with lower 
gastrointestinal bleeding send to a hospital for their study. Value of clinical data in predicting normal or pathological findings. Rev Esp Enferm Dig. 1996;88(1):16-25.

13. Hreinsson JP, Egisdottir $S$ and Bjornsson ES. Acute lower gastrointestinal bleeding: A population-based five-year follow-up study. United European Gastroenterology Journal. 2019;7(10):1330-1336.

https://doi.org/10.1177/2050640619863517

14. Strate LL and Gralnek IM. Management of Patients with Acute Lower Gastrointestinal Bleeding. Am J Gastroenterol. 2016; 111(4):459-474.

https://doi.org/10.1038/ajg.2016.41
15. Gralnek IM, Neeman $Z$ and Strate LL. Acute Lower Gastrointestinal Bleeding. N Engl J Med. 2017; 376:1054-1063. https://doi.org/10.1056/NEJMcp1603455

16. Alobaidi QA, Al-shammari AJ and Al- Faham AM. Colonoscopy finding of Lower Gastrointestinal Bleeding (LIGB) in Al Seder Medical City (in Al-Najaf)/Iraq. J Pharm Sci \& Res. 2018;10(4):839-842.

17. Shrestha UK. Etiological profile, gender difference and age group patterns of 415 patients presenting with lower gastrointestinal bleeding in the western region of Nepal. Journal of Advances in Internal Medicine. 2014;03(02):52-55.

https://doi.org/10.3126/jaim.v3i2.14064

\section{Authors Contribution:}

SB -Concept and design of the study, data collection, interpreted the results, prepared first draft of manuscript and critical revision of the manuscript; RRA- Data collection, reviewed the literature and manuscript preparation; BJ - Design of the study, preparation of manuscript and revision of the manuscript; DK - Data collection, preparation of manuscript and revision of the manuscript

\section{Work attributed to:}

Unit of Medical Gastroenterology, Department of Medicine, Manipal Teaching Hospital, Pokhara, Nepal.

\section{Orcid ID:}

Dr. Subash Bhattarai - (D) https://orcid.org/0000-0001-7428-7460

Dr. Ramesh Raj Acharya- (1) https://orcid.org/0000-0002-1373-5642

Dr. Bishnu Jwarchan- (D) https://orcid.org/0000-0001-5190-5975

Dr. Dipesh Karki- (i) https://orcid.org/0000-0002-4789-8765

Source of support: None, Conflicts of Interest: None 